\title{
Ethische Abwägungen beim Infektionsschutz
}

\author{
Christiane Woopen ${ }^{(\bowtie)}$ \\ Forschungsstelle Ethik, Universität zu Köln, Köln, Deutschland \\ christiane.woopen@uni-koeln.de
}

Schlüsselwörter: COVID-19 · Infektionsschutz · Ethische Güter ·

Güterabwägung

Der Vortrag musste aus organisatorischen Gründen am Tag selbst spontan erheblich gekürzt werden und sprach nur wenige Aspekte für die folgende Podiumsdiskussion an.

\section{Kurze Theorie der Güterabwägung}

Bei Infektionsschutzmaßnahmen können die unterschiedlichsten ethischen Güter (Abb. 1) miteinander in Konflikt geraten. Ein plakatives Beispiel: Wenn es um Schulschließungen geht, sind die Güter Gesundheit und Leben in eine Waagschale zu werfen und die Güter Bildung, soziale Gerechtigkeit und Solidarität in die andere. 


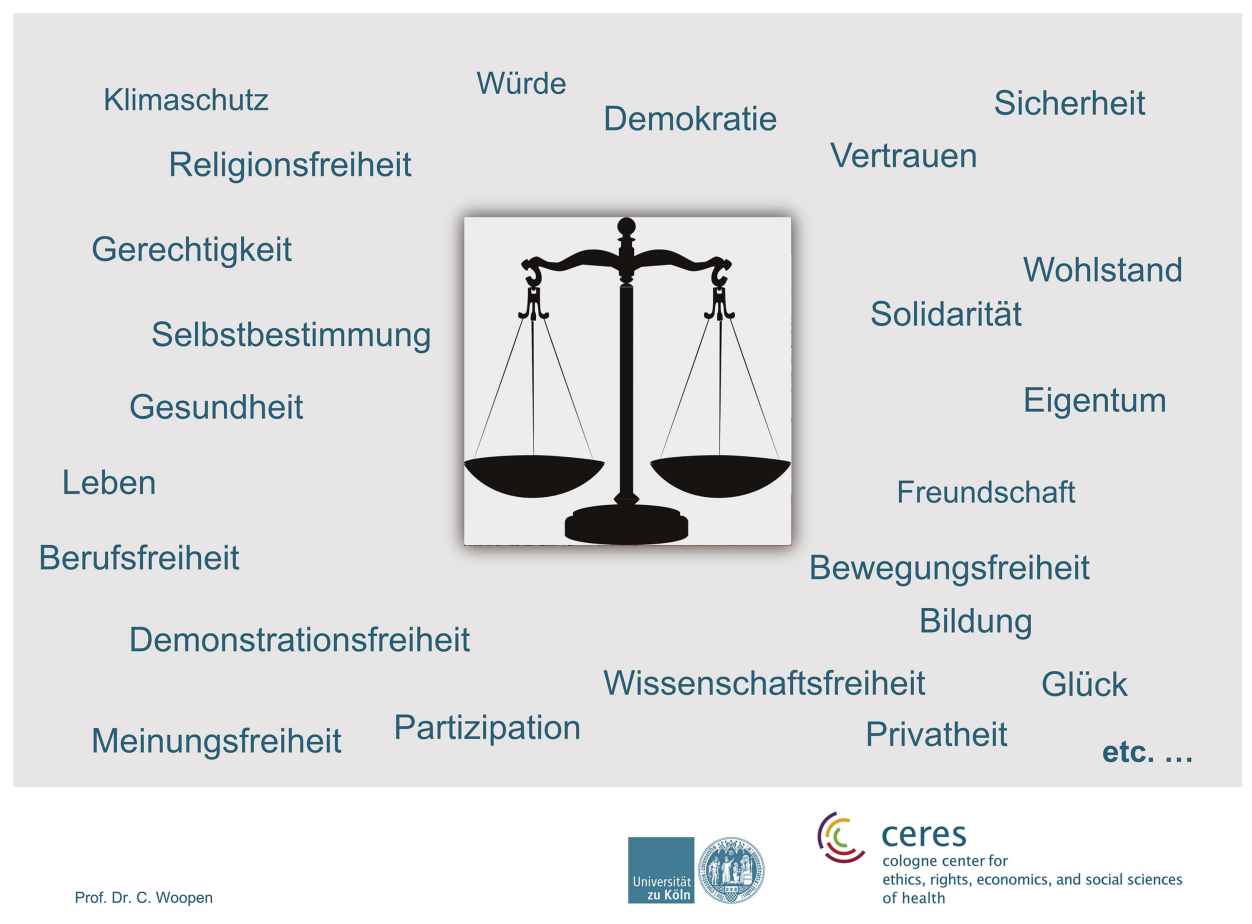

Abb. 1 Beispielhafte Güter, die abzuwägen sind

Welches Gut hat mehr Gewicht? Gibt es ein höchstes Gut? Diese grundsätzlichen Fragen sind nicht nur in der breiten Öffentlichkeit, sondern auch unter Ethikern, Juristen und Philosophen umstritten.

Manche ethischen Theorien setzen Güter in eine hierarchische Ordnung und legen im Einklang mit der deutschen Verfassung die Würde des Menschen als höchstes und nicht abwägbares Gut zugrunde. Die folgende Hierarchisierung, die ebenfalls diesen Ausgangspunkt wählt, ist gleichwohl offen für verschiedene Vorstellungen davon, was individuell als sinnvolles Leben angesehen wird. Demnach gibt es besondere Güter, wie Leben, Gesundheit, Freiheit und Selbstbestimmung, ohne die ein sinnvolles Leben nicht verwirklicht werden kann, weil keine Handlungsmöglichkeit oder -freiheit besteht. Diese Güter kann man in der Philosophie als transzendentale Güter erster Ordnung bezeichnen, also als Güter, die die Bedingung der Möglichkeit von etwas sind - in diesem Fall die Bedingungen von biologischem Leben und von Freiheit. Weiterhin gibt es Güter, die den Erfolg von Lebensplänen ermöglichen, dazu zählt etwa die Bildung oder die Selbstachtung. Diese werden als transzendentale Güter zweiter Ordnung bezeichnet. Schließlich gibt es noch Güter dritter Ordnung, auch instrumentelle Güter genannt: Darunter versteht man Güter, die man als Mittel zur Verwirklichung lediglich situativ angestrebter Ziele benötigt [1].

Bei der Güterabwägung muss die Ordnung, also der Rang eines Gutes, in Betracht gezogen werden. Allerdings reicht das nicht schon aus. So stehen sich etwa nicht 
immer ein höherrangiges und ein niederrangiges Gut gegenüber, sondern zwei gleichrangige, etwa Leben gegen Leben. Zum Beispiel lässt sich derzeit eine Übersterblichkeit nicht nur bei den COVID-19 Erkrankten beobachten, sondern es gibt auch eine Übersterblichkeit bei anderen Erkrankungen. Dies ist unter anderem darauf zurückzuführen, dass die gesundheitliche Versorgung dieser Erkrankten nicht auf dem gleichen Niveau gewährleistet ist wie sonst: Manche Erkrankte gehen aus Angst vor einer COVID-19-Erkrankung nicht zum Arzt, Praxen werden geschlossen und Behandlungen von Patienten, die nicht an COVID-19 leiden, werden verschoben. Mit anderen Worten: Durch die Infektionsschutzmaßnahmen sterben auch Menschen, die möglichweise sonst nicht gestorben wären, etwa an einem Herzinfarkt, einem Schlaganfall, an Krebs oder an Einsamkeit und Trauer. Das muss man in die Abwägung der Infektionsschutzmaßnahmen einbeziehen.

\section{Güterabwägung bei einzelnen Maßnahmen}

Abstand halten. Bei der Maßnahme „Abstand halten“ ist der Schutz der öffentlichen Gesundheit mit Gütern wie Lebensqualität, soziale Nähe und körperliche Zuwendung abzuwägen. Diese Güter sind gemeinhin nicht als höchstrangige Güter anzusehen. Es gibt aber Menschen, die nur dann ins Handeln und in die eigene Selbstverwirklichung kommen, wenn sie körperlich unterstützt werden: beispielsweise Bewohner in Altenheimen oder Menschen mit bestimmten Erkrankungen. Ethisch betrachtet ist für diese Menschen körperliche Nähe ein Gut erster Ordnung, denn es ermöglicht das Handeln im Hinblick auf ein gelingendes Leben, zuweilen sogar auf das Überleben.

Maskenpflicht. Bei der Verpflichtung zum Tragen einer Alltagsmaske gibt es gegenwärtig, im Oktober 2020, keinen nennenswerten Eingriff in ein höherrangiges Gut. Wenn jedoch Impfstoffe verfügbar werden und die Impfungen anlaufen, wird die Maskenpflicht zu einem Konflikt führen. Dann werden Menschen Fragen stellen wie: „Wenn ich doch jetzt geimpft bin und keinen anstecke, warum soll ich dann jetzt noch eine Maske tragen?" Sie werden Masken als gar nicht mehr erforderlich empfinden weder dafür, dass sie sich selbst schützen, noch dafür, dass sie andere schützen, die Tragepflicht als Eingriff in ihre Freiheiten empfinden und auf die Wiederherstellung ihrer grundrechtlich verbürgten Freiheiten pochen. Allerdings haben andere Redner auf dieser Tagung bereits dargelegt, dass man noch nicht weiß, ob nach Impfungen auch keine Ansteckung mehr erfolgen kann. Also muss man die Menschen davon überzeugen, dass die Tragepflicht weiterhin sinnvoll und geboten ist, zumindest um andere zu schützen. Hier kann man auch an die Solidarität appellieren.

Corona-Warn-App. Bei der Corona-Warn-App hat Deutschland aus Gründen des Datenschutzes eine Lösung gewählt, die den Nachteil hat, dass es keine Übersicht über die epidemiologische Dynamik einschließlich der risikoreichsten Orte gibt. Derzeit lässt sich nur ein Viertel der insgesamt gemeldeten Fälle einem Infektionsort zuordnen, also weiß man bei drei Vierteln nicht Bescheid [2]. Aus der ethischen Perspektive muss man sagen, dass der Schutz der Privatheit nicht von vorneherein höherrangiger ist als der Infektionsschutz für die hochrangigen Güter von Leben 
und Gesundheit. Und wenn man eine Information, die für den Zuschnitt infektionseindämmender Maßnahmen entscheidend ist, nicht kennt, so sollte man eine ganze Menge unternehmen, um diese Wissenslücke zu schließen. Insofern wäre hier noch einmal darüber nachzudenken, wie wir die Privatsphäre bestmöglich schützen können, aber trotzdem diese Information bekommen: $\mathrm{Zu}$ denken ist etwa an das gesetzliche Verbot, die Information für andere Zwecke zu verwerten, oder an den Einsatz von Datentreuhändern, die in regulierter Weise tätig werden können. ${ }^{1}$

Teststrategien. Derzeit verfolgt Deutschland eine reaktive Teststrategie. Aus der ethischen Perspektive heraus kann und sollte man sich auch eine andere, präventive Teststrategie vorstellen. Es wäre durchaus möglich, die Testkapazitäten - sowohl PCR-Tests als auch Schnelltests bis hin zu selbst anzuwendenden Schnelltests - so weiterzuentwickeln und zu skalieren, dass die gesamte Bevölkerung mehrmals in der Woche über einige Monate hinweg getestet werden kann. So erhielte man ein zuverlässiges Gesamtbild der pandemischen Lage und könnte gezielter eingreifen sowie Infektionsketten verhindern als bisher. Das wäre eine Dauerstrategie und möglicherweise auch für eine nächste Pandemie hilfreich, die - auch nach Aussagen der Experten auf dieser Tagung - auf uns zukommen wird.

Einschränkung des Freizeitbereichs. Die deutsche Politik hat derzeit entschieden, dass die Bereiche von Freiheit und Kultur hinter Wirtschaft und Bildung zurückstehen sollen. Wenn man so verfährt, sollte man offen sagen, warum die Kultur so viel weniger wert ist als die Wirtschaft und die Bildung. Selbstverständlich sichert die Wirtschaft unseren Wohlstand und die Sozialsysteme. Andererseits gehören Museen, Kinos und Theater auch zur Bildung, und wirtschaftlich hängen viele Existenzen daran. Vor allem aber ist die Kultur auch Reflexionsraum, Spiegelung und Kit der Gesellschaft. Insofern sind hier ethisch sehr relevante Entscheidungen getroffen worden, die nicht evidenzgestützt sind, weil man viele wesentliche Daten zur Infektionsübertragung in Einrichtungen, die gute Hygiene-Konzepte eingeführt haben, überhaupt nicht kennt.

\section{Gesundheit versus Wirtschaft}

Immer wieder wird gefragt: Ist die Wirtschaft wichtiger als die Gesundheit oder ist die Gesundheit wichtiger als die Wirtschaft? Um das zu beantworten, hilft es, sich zunächst einer anderen Frage zuzuwenden: Wofür sind Wirtschaft und Wohlstand da? Sie unterstützen den Menschen in seinem Leben, können zu einem gelingenden sozialen und individuellen Leben beitragen und auch Auswirkungen auf die Gesundheit haben. Aber sie sind nicht so fundamental handlungsermöglichend wie das Leben selbst, sondern erweitern lediglich das Spektrum der Handlungsmöglichkeiten. Es geht also um ein transzendentales Gut zweiter Ordnung. Oder anders ausgedrückt: Die Wirtschaft ist immer Mittel zum Zweck. Andererseits sind die Gesundheit und

\footnotetext{
$\overline{1 \text { s. dazu auch [3] }}$
} 
das Leben von hypothetischen Personen in der Zukunft - es geht hier um statistische Modelle und epidemiologische Sachverhalte - nicht automatisch mehr wert als die kollektive Wertschöpfung und das Gemeinwohl.

\section{Literatur}

1. Woopen, C.: Selektion aufgrund genetischer Diagnostik? Handlungstheoretisch fundierte Güterethik und ihre Anwendung am Beispiel der Präimplantationsdiagnostik. In: Honnefelder, L.; Streffer, C. (Hrsg.) Jahrbuch für Wissenschaft und Ethik, Bd. 10, S. 343353. de Gruyter, Berlin, New York (2005)

2. https://www.rki.de/DE/Content/InfAZ/N/Neuartiges_Coronavirus/Situationsberichte/ Okt_2020/2020-10-27-de.pdf. Zugegrifen: 31. Jan. 2021

3. Datenethikkommission: Gutachten für die Bundesregierung. https://www.bmi.bund.de/ SharedDocs/downloads/DE/publikationen/themen/it-digitalpolitik/gutachten-datenethikkommission.html. Zugegrifen: 31. Jan. 2021

Open Access Dieses Kapitel wird unter der Creative Commons Namensnennung - Nicht kommerziell - Keine Bearbeitung 4.0 International Lizenz (http://creativecommons.org/ licenses/by-nc-nd/4.0/deed.de) veröffentlicht, welche die nicht-kommerzielle Nutzung, Vervielfältigung, Verbreitung und Wiedergabe in jeglichem Medium und Format erlaubt, sofern Sie den/die ursprünglichen Autor(en) und die Quelle ordnungsgemäß nennen, einen Link zur Creative Commons Lizenz beifügen und angeben, ob Änderungen vorgenommen wurden. Die Lizenz gibt Ihnen nicht das Recht, bearbeitete oder sonst wie umgestaltete Fassungen dieses Werkes zu verbreiten oder öffentlich wiederzugeben.

Die in diesem Kapitel enthaltenen Bilder und sonstiges Drittmaterial unterliegen ebenfalls der genannten Creative Commons Lizenz, sofern sich aus der Abbildungslegende nichts anderes ergibt. Sofern das betreffende Material nicht unter der genannten Creative Commons Lizenz steht und die betreffende Handlung nicht nach gesetzlichen Vorschriften erlaubt ist, ist auch für die oben aufgeführten nicht-kommerziellen Weiterverwendungen des Materials die Einwilligung des jeweiligen Rechteinhabers einzuholen. 